\title{
Nanostructure and EELS Characterization of Diluted Magnetic Semiconductor $\mathrm{Zn}_{1-\mathrm{x}} \mathrm{Co}_{\mathrm{x}} \mathrm{O}$ Nanoneedles
}

\author{
W.L. Zhou ${ }^{*}$, J.J. Chen, K. Sun ${ }^{* *}$, and L.M. Wang ${ }^{* *}$ \\ *Advanced Materials Research Institute, University of New Orleans, New Orleans, LA 70148 \\ ** Dept. of Nuclear Engineering \& Radiological Sciences, University of Michigan, Ann Arbor, \\ MI 48109
}

In recent years, diluted magnetic semiconductors (DMSs) have stimulated much interest because of their promising applications in spintronic devices, such as spin-valve transistors, spin lightemitting diodes, non-volatile memory, etc [1,2] Therefore, ferromagnetic DMSs with Curie temperature $\left(T_{\mathrm{C}}\right)$ higher than room temperature are crucial for practical device applications. In this paper, characterization of Co-doped $\mathrm{ZnO}$ nanoneedles with room temperature ferromagnetic ordering fabricated through pulsed laser deposition (PLD) method is presented.

Co-doped $\mathrm{ZnO}$ nanoneedles were formed on silicon (100) substrate by PLD [3]. The morphology of as-synthesized $\mathrm{Zn}_{0.95} \mathrm{Co}_{0.05} \mathrm{O}$ nanoneedles was investigated by Carl Zeiss 1530 VP field-emission scanning electron microscope (FESEM). Nanostructures of the nanoneedles were determined by JEOL 2010 transmission electron microscope (TEM) equipped with EDAX DXPrime electron energy dispersive X-ray analysis (EDS). Electron energy loss spectroscopy (EELS) and energy filter TEM (EFTEM) were obtained using a JEOL 2010F STEM/TEM equipped with Gatan imaging filter (GIF). X-ray diffraction (XRD) was done by Philips X'Pert MPD using a CuK source.

Fig. 1(a) shows a typical FESEM image of the Co-doped $\mathrm{ZnO}$ nanoneedles. The nanoneedles have a growth direction preferentially perpendicular to Si substrate, which is confirmed by X-ray diffraction in Fig. 1(b). Fig. 1(d) is a high-resolution electron microscopy (HREM) image of $\mathrm{ZnO}$ nanoneedle along [2 110 ] zone axis, highlighted by the upper rectangular area of Fig. 1(c). A representative surface structure with stacking faults (the lower rectangular area of Fig. 1(c)) is shown in Fig. 1(e), and its back and forth fast Fourier transformation (FFT) image using the $000 \pm 1$ diffraction spots is displayed in Fig. 1(f). By positioning the nanoprobe along $c$-axis of a nanoneedle (Fig. 2(a)), Co L 2,3 peaks with similar intensity were obtained as shown in Fig.2 (b). EELS mapping observation also shows that cobalt homogeneously distributes along the nanoneedles. Magnetic measurement shows room temperature ferromagnetism was achieved, which is attributed to the successfully doping of $\mathrm{Co}$ into the $\mathrm{ZnO}$ nanoneedles.

\section{References}

[1] J. K. Furdyna, J. Appl. Phys. 64, R29 (1988).

[2] S. J. Pearton, W. H. Heo, M. Ivill, D. P. Norton, and T. Steiner, Semicond. Sci. Technol. 19, R59 (2004).

[3] J.J. Chen, M.H. Yu, W.L. Zhou, K. Sun, and L.M. Wang, Appl. Phys. Lett. 87, 173119 (2005).

[4] This work was supported by the DARPA Grant No.HR0011-04-1-0029 and a research grant from Louisiana Board of Regents Contract No.LEQSF (2003-06)-RD-B-13. 

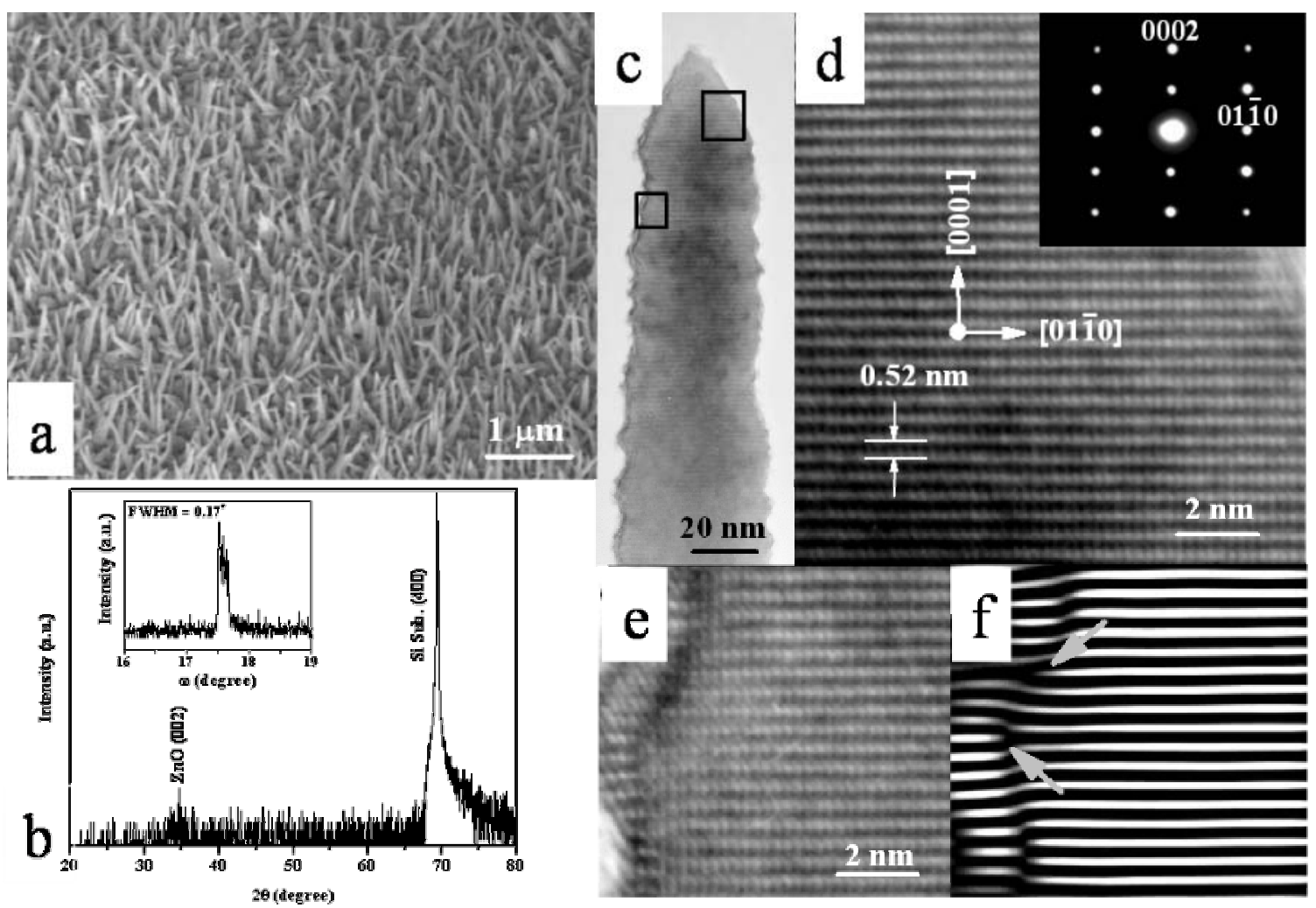

Fig. 1 (a) FESEM image of Co-doped ZnO nanoneedle. (b) X-ray diffraction pattern. (c) A single Co-doped nanoneedle. (d) HREM image of upper rectangular area and corresponding SAD pattern (inset), showing $c$-axis growth. (e) HREM image of lower rectangular area, showing typical stacking faults near the nanoneedle surface. (f) The back and forth fast Fourier transformation (FFT) image of (e) by using the $000 \pm 1$ spots.
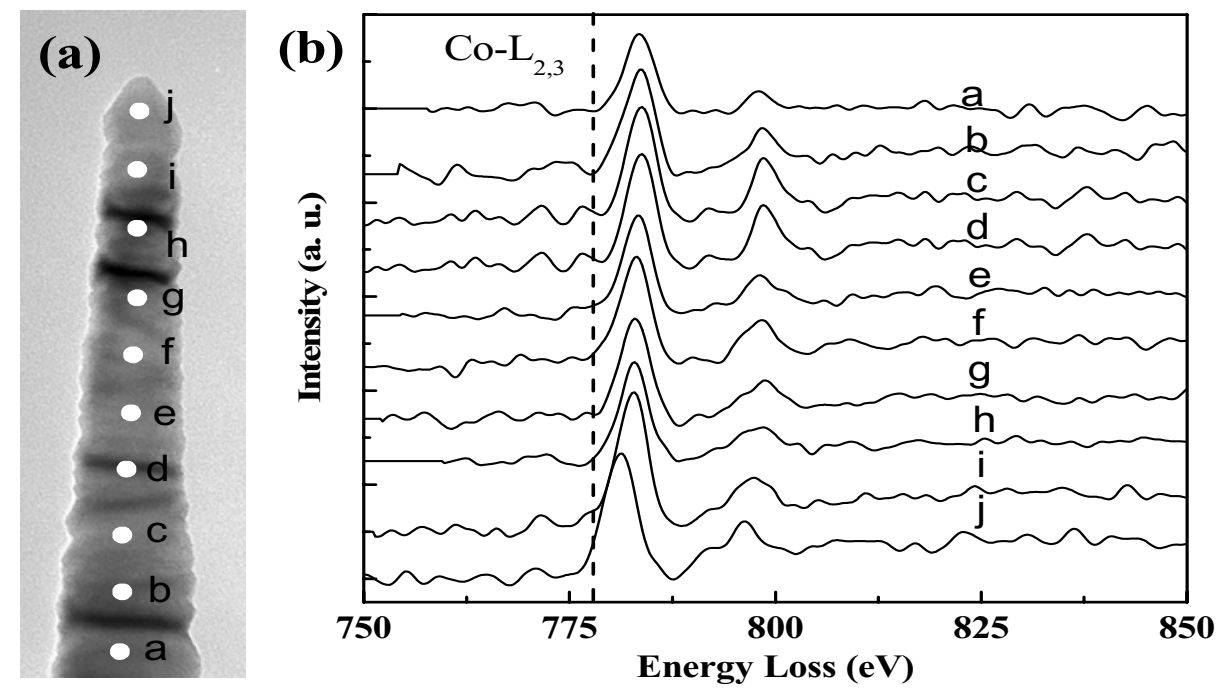

Fig.2 (a) TEM image showing the probe positions along the growth direction of the nanoneedle for EELS measurement and (b) corresponding EELS spectra. The Co- $\mathrm{L}_{2,3}$ edge is shown by the dashed line. 